ARTICLE

\title{
Comparing breast and lung cancer patients' experiences at a UK Cancer Centre: implications for improving care and moves towards a person centered model of clinical practice
}

\author{
Elizabeth A Davies PhD FFPH ${ }^{a}$, Peter Madden BS, MSc ${ }^{b}$, Victoria H Coupland BSc MPH , $^{\mathrm{c}}$ \\ Mairead Griffin MA, BSc, RN ${ }^{\mathrm{d}}$ and Alison Richardson BN RGN MSc, PhD ${ }^{\mathrm{e}}$ \\ a Medical Director and Reader in Cancer and Public Health, King's College London UK, Thames Cancer \\ Registry, London. UK \\ b Researcher and Statistician, King's College London UK, Thames Cancer Registry, London. UK \\ c Information Analyst and Researcher, King's College London UK, Thames Cancer Registry, London. UK \\ d Head of Nursing and Lead Cancer Nurse, Guy's and St Thomas' NHS Foundation Trust, London, UK \\ e Clinical Professor of Cancer Nursing and End of Life Care, University of Southampton \& Southampton \\ University Hospitals NHS Trust, School of Health Sciences, Southampton, UK
}

\begin{abstract}
Patient surveys are increasingly used to obtain feedback about experiences of care to guide changes in the way services are delivered. We compared the experiences of breast and lung cancer patients at diagnosis, in-patient and outpatient care at a Cancer Centre using a locally adapted version of the Picker Institute Cancer Patient Experience Survey. 65\% of breast (82/127) and 65\% of lung (75/116) cancer patients responded. Both groups reported good experiences at diagnosis, good support from clinical nurse specialists, and had very positive overall views. Breast patients had slightly poorer experiences of hospital stays while lung patients less often received clinical nurse specialist support and information about other support. Both groups reported that written information was not always provided. 84\% (132/157) consented to data on the clinical severity of their condition being used to investigate whether this influenced experiences. This study showed that supportive care policies are being implemented at diagnosis and patients value clinical nurse specialist care. Sufficiently large comparative surveys can identify specific areas of performance for services to focus upon in order to improve quality of care. Patients facing poor prognoses can complete surveys and future studies might explore whether disease stage is associated with differing experiences.
\end{abstract}

\section{Keywords}

Patient-centred care, patient experience, surveys, breast cancer, lung cancer

\section{Correspondence Address}

Dr. Elizabeth A Davies, Thames Cancer Registry, 42 Weston Street, London SE1 3QD, UK.

E-mail: Elizabeth.Davies@kcl.ac.uk

Accepted for publication: 31 January 2011 Funding: Guy’s and St Thomas’s Charity

\section{Introduction}

There is increasing international interest in using information on patients' experiences of care to guide changes in the way services might be organised and delivered [1,2]. Patient surveys are one means of collecting feedback that can be used to monitor the extent to which care is patient-centred and to plan and focus quality improvement efforts. In England, two national surveys of patients' experiences of cancer services have reported, the first undertaken in $1999-2000$ by the National Patient
Survey Programme [3] and the second in 2004 by the National Audit Office [4]. Both surveys found that patients with breast cancer reported the best experiences and that this persisted after adjustment for age, region, and deprivation of area of residence. Patients in London, however, tended to report worse experience. The second survey showed some national improvement in experience, particularly in the areas of information, communication and trust in health professionals [4]. A later analysis suggested that the experiences of London breast cancer patients had declined in some areas of care [5]. A further national survey has just been carried out in 2010 for 158 
English hospital trusts and this exercise may be repeated annually [6,7].

As well as information on experiences of services, surveys can provide information about how clinical aspects of patients' conditions affect the quality of their everyday life and other patient-reported outcomes [8,9]. In England the intention is that data on patients' experiences of NHS cancer care will be reported along side a range of patient outcomes for particular geographical areas or services $[6,10]$. So far little research has considered whether cancer patients' experiences and their outcomes are directly related [11], although studies in the US have begun to link cancer registry data on diagnosis to patient survey data on quality of life and disability [12]. Commonly raised concerns about using patient survey data to guide quality improvement include how representative patients responding are, whether the more ill patients with a poor outlook are excluded from receiving or completing questionnaires, the possibility that responses may be influenced by disease severity or a poor prognosis, and lack of specific results for large enough groups of patient looked after by particular services $[13,14]$.

The 'Patient Centred Care' project for the Integrated Cancer Centre for South East London (comprising the NHS Foundation Trusts of Guy's and St Thomas' and King's College Hospitals) seeks to improve patient and staff experiences of breast and lung cancer services. As part of this project we carried out a survey of patients' experiences with the aims of:

1) Comparing experiences reported by breast and lung cancer of diagnosis, in-patient and out-patient care

2) Suggesting improvements to services to improve the experience of patients at each stage of their care

3) Determining the feasibility of surveying patients facing poor prognoses and of obtaining patient consent to link experience data with that on disease severity.

\section{Methods \\ Developing and piloting the questionnaire}

We adapted a questionnaire recently used in North London [15], and based upon earlier national surveys undertaken by the Picker Institute [16]. To ensure questions were relevant to local services we invited comments from 26 multi-disciplinary staff within the Integrated Cancer Centre. Additional questions concerning contact with a "key worker" to address cancer peer review standards [17], and about disability and long term conditions were suggested. We decided to use the word cancer in the questionnaire because all patients would have been informed of their diagnosis, and not to exclude any patients at the outset from the survey. We focussed on making questions as specific as possible, both about areas of care and about hospital site so that they would provide information useful for quality improvement. We decided on 102 questions organised into six sections: A) Finding out what was wrong with you (31) B) Outpatient treatment and appointments (18) C) Recent overnight or day case stay (26), D) Leaving hospital (10) E) Clinical nurse specialist care (6) and F) Overall views about care (11). Two final open questions asked patients what they thought was particularly good about their care and what areas they thought could be improved. We also asked patients for consent to obtain anonymous information from their clinical team on the clinical severity of their condition to investigate whether this was related to experiences of care. The adapted questionnaire was piloted by post with six patients (three breast and three lung), and in person with 11 patients (six breast and five lung) attending clinic. The 14 patients responding were overwhelmingly positive about the focus of the questionnaire. Despite some concern from staff about the burden of completing the questionnaire, no patient complained about its length, but some changes to wording and layout were suggested.

\section{Ethical and research and development approval}

The National Research Ethics Service advised that the survey was service evaluation as opposed to research, and thus approval from an ethics committee was not necessary. Research and Development service evaluation approval was obtained from each hospital.

\section{Identifying participants for the survey}

Based on previous analyses we determined that to compare experiences at a hospital site we needed ideally to recruit 50 respondents with each cancer (3). Contact details and NHS numbers of patients who had invasive breast or lung cancer diagnosed in 2008 and 2009 were obtained from hospital databases, duplicate cases removed and vital status and addresses checked using the NHS Strategic Tracing Service. The final sample included 127 breast cancer patients who received their first definitive treatment at GST between October 2008 and April 2009 and 116 lung cancer patients who received their first definitive treatment between January and April 2009. The tighter time frame was used to select lung cancer patients because their poor outlook meant we needed to ensure they would be well enough to complete a questionnaire. 37 breast and 69 lung cancer patients were also identified from $\mathrm{KCH}$ databases, but we report only on GST patients because they represented a large enough sample to compare experiences at the same hospital.

\section{Sending the survey and offering help with its completion}

Our approach to patients was informed by a Cochrane review on increasing questionnaire response rates which 
found that personalised letters, stamped return envelopes, coloured print and an assurance of confidentiality and first class outward mailing were successful strategies ${ }^{18}$. Patients received survey packs during July and August 2009 which included a questionnaire, an information sheet, and a letter from the lead cancer nurse (MG) explaining that the survey was voluntary and confidential and that results would be used to improve services. A short summary of the project, translated into 10 languages (Arabic, Bengali, Chinese, French, Polish, Portuguese, Spanish, Somali, Turkish and Vietnamese), explained how to receive help from the hospital translation service. The researcher's telephone number was provided for patients who needed more help or explanation to fill in the questionnaire and numbers for nurse consultants for those who wished to discuss any concerns it raised about their illness or treatment. Patients who did not reply were sent two reminders, after re-checking their vital status. Six lung cancer patients were excluded at this point.

\section{Analysis}

We focus on reporting comparative results where at least 40 breast and lung patients responded to the same questions. Results are reported as percentages for experiences in each area of care, excluding missing values. We also present these as bar charts highlighting where less than $75 \%$ of patients reported a good experience. Responses to the open questions were typed in full, collated, compared and sorted into themes to determine where they added new information about suggested improvements.

\section{Results \\ Response to the survey}

$65 \%(82 / 127)$ of breast and 65\% (75/116) of lung patients returned completed questionnaires. No patient requested translation services, but three breast and four lung patients rang the researcher to ask which parts of their care were relevant to the questionnaire and two lung patients requested help completing it. Two patients requested more support with their condition and were referred to a nurse consultant for further help. A high proportion of breast (66\%, 54/82) and lung patients (65\%, 56/75) added comments about their care and suggestions for improving services.

\section{Characteristics of patients}

Table 1 shows demographic and clinical characteristics of patients responding. Patients with breast cancer were more commonly from ethnic groups other than white (24\%, 20/82 compared to 5\%, 4/75), lived in London (95\%, 78/82 compared to $51 \%, 38 / 75)$ and in more deprived areas (70\%, 57/82 compared to $44 \%, 33 / 75$ ) and less likely to have had surgery as their only treatment (12\%, 10/82 compared to 51\%, 38/75). These differences were significant $(\mathrm{p}<0.01)$. In breast cancer non-responders did not differ by age, but were less likely to have had chemotherapy, more likely to have had surgery and slightly less likely to live in deprived areas. In lung cancer nonresponders did not differ by age but were slightly less likely to have had surgery (data not shown).

Table 1 Characteristics of patients responding to the survey at the Cancer Centre

Figures are numbers (percentages) unless otherwise stated

\begin{tabular}{|c|c|c|c|c|}
\hline \multirow{2}{*}{$\begin{array}{l}\text { Age } \\
\text { Mean (range) }\end{array}$} & \multicolumn{2}{|c|}{ Breast $(n=82)$} & \multicolumn{2}{|c|}{ Lung ( $n=75)$} \\
\hline & 62 & $\begin{array}{l}28- \\
94\end{array}$ & 68 & $22-87$ \\
\hline $\begin{array}{l}\text { Ethnicity } \\
\text { White } \\
\text { Black } \\
\text { Asian } \\
\text { Mixed } \\
\text { Other } \\
\text { Missing } \\
\text { Total }\end{array}$ & $\begin{array}{c}62 \\
13 \\
4 \\
2 \\
1 \\
0 \\
82\end{array}$ & $\begin{array}{l}(76) \\
(16) \\
(5) \\
(2) \\
(1) \\
(0) \\
(100)\end{array}$ & $\begin{array}{c}70 \\
1 \\
2 \\
0 \\
1 \\
1 \\
75\end{array}$ & $\begin{array}{l}(93) \\
(1) \\
(3) \\
(0) \\
(0) \\
(1) \\
(100)\end{array}$ \\
\hline $\begin{array}{l}\text { Deprivation quintile } \\
1 \text { (Most affluent) } \\
2 \\
3 \\
4 \\
5 \text { (Most deprived) } \\
\text { Total }\end{array}$ & $\begin{array}{c}6 \\
4 \\
15 \\
28 \\
29 \\
82\end{array}$ & $\begin{array}{l}(7) \\
(5) \\
(18) \\
(34) \\
(35) \\
(100)\end{array}$ & $\begin{array}{c}9 \\
21 \\
12 \\
16 \\
17 \\
75\end{array}$ & $\begin{array}{l}(12) \\
(28) \\
(16) \\
(21) \\
(23) \\
(100)\end{array}$ \\
\hline $\begin{array}{l}\text { Place of residence } \\
\text { London } \\
\text { South East England } \\
\text { Elsewhere } \\
\text { Total }\end{array}$ & $\begin{array}{c}78 \\
4 \\
0 \\
82\end{array}$ & $\begin{array}{c}(95) \\
(5) \\
(0) \\
(100)\end{array}$ & $\begin{array}{c}38 \\
37 \\
0 \\
75\end{array}$ & $\begin{array}{c}(51) \\
(49) \\
(0) \\
(100)\end{array}$ \\
\hline Disability or long term & condit & & & \\
\hline $\begin{array}{l}\text { Yes } \\
\text { Mobility } \\
\text { Blind or partially } \\
\text { sighted } \\
\text { Deaf or hearing } \\
\text { impaired } \\
\text { Communication } \\
\text { Learning difficulty } \\
\text { Mental health } \\
\text { condition } \\
\text { Other } \\
\text { Nil } \\
\text { Prefer not to } \\
\text { say/missing }\end{array}$ & $\begin{array}{c}7 \\
0 \\
1 \\
3 \\
0 \\
48 \\
5\end{array}$ & $\begin{array}{l}(35) \\
(24) \\
(2) \\
(9) \\
(0) \\
(1) \\
(4) \\
(0) \\
(59) \\
(6)\end{array}$ & $\begin{array}{c}36 \\
23 \\
0 \\
6 \\
1 \\
0 \\
0 \\
12 \\
37 \\
2\end{array}$ & $\begin{array}{l}(48) \\
(31) \\
(0) \\
(8) \\
(1) \\
(0) \\
(0) \\
(16) \\
(49) \\
(3)\end{array}$ \\
\hline $\begin{array}{l}\text { Year of Diagnosis } \\
2007-2008 \\
2009 \\
\text { Missing } \\
\text { Total }\end{array}$ & $\begin{array}{c}46 \\
30 \\
6 \\
82\end{array}$ & $\begin{array}{c}(56) \\
(37) \\
(7) \\
(100)\end{array}$ & $\begin{array}{c}29 \\
41 \\
5 \\
75\end{array}$ & $\begin{array}{c}(39) \\
(55) \\
(7) \\
(100)\end{array}$ \\
\hline $\begin{array}{l}\text { Treatment } \\
\text { Surgery only } \\
\text { Surgery and other } \\
\text { treatment } \\
\text { Other treatment only } \\
\text { No treatment yet } \\
\text { Missing } \\
\text { Total }\end{array}$ & $\begin{array}{c}10 \\
5 \\
0 \\
82\end{array}$ & $\begin{array}{l}(12) \\
(70) \\
(12) \\
(6) \\
(0) \\
(100)\end{array}$ & $\begin{array}{c}26 \\
2 \\
2 \\
75\end{array}$ & $\begin{array}{l}(51) \\
(9) \\
(35) \\
(3) \\
(3) \\
(100)\end{array}$ \\
\hline
\end{tabular}

${ }^{1}$ From the Indices of Multiple Deprivation 2007

${ }^{2}$ Categories for specific disability or condition are not exclusive 


\section{Comparing the experiences of breast and lung cancer patients}

\section{A. Finding out what was wrong with you}

All but three patients reported they had been told their diagnosis in person. The first four findings of Figure 1 show very high positive experiences about communication of the diagnosis, with breast cancer patients reporting slightly more positive experiences than lung cancer patients. $80 \%$ or more of both breast and lung patients reported they had understood the explanation about their condition, been told about it with sensitivity and care, had their questions answered and been involved. $75 \%$ had understood explanations about treatment. The clinical nurse specialist was not always present for these consultations but more commonly so for breast (65\%, $53 / 82)$ than lung $(23 \%, 17 / 74)$ patients. A partner /spouse or family member was less often present for breast (56\% 46/82) than for lung patients (65\%, 48/74).

Patients, however, varied in whom they said they would have wanted to be present, with 39\% (32/82) of breast and 50\% (37/74) of lung patients saying that at the time they would not have wanted a family member, friend or nurse present. No patient received a tape recording of their diagnosis discussion, around one third of each group reported being given a written record of the discussion, but higher proportions of breast $(77 \%, 62 / 81)$ than lung patients $(58 \%, 42 / 73)$ reported being given written or printed information about their condition or treatment. Slightly lower proportions (68\% (54/79) of breast and 66\% (48/73) of lung patients) reported receiving a copy of the letter sent to their general practitioner. Although high proportions were given the name of a key worker to contact about any concerns they had, this was more commonly the case for breast $(93 \%, 75 / 81)$ than lung patients (75\%, 53/71).

\section{B. Out-patient treatment and appointments}

Figure 2 shows the similar overall positive experience of lung and breast cancer patients at their most recent outpatient and treatment appointments with at least $75 \%$ reporting good experiences of the way they were treated and of explanations during their consultations. Differences of experiences between the two patient groups were minor. Similar proportions (74\%, 55/74 of breast and 69\%, 29/42 of lung) reported being given the option of receiving copies of letters sent to their general practitioner. However, fewer patients (56\%, 42/75 of breast and 47\%, 20/43) of lung) reported being given written or printed information about their condition or treatment.

\section{Most recent overnight or day case stays}

$65 \%(53 / 81)$ of breast and $68 \%$ (46/68) of lung patients reported an overnight or day case stay in the last six months. Figures 3a and b show that experiences during these stays were generally good, although overall lung cancer patients reported better experience. Breast patients were less likely to report that there were enough nurses on duty (67\%, 34/51 compared to $86 \% 38 / 44)$, that family and friends were involved in decisions about treatment $(47 \%$, $25 / 52$ compared to $59 \%, 26 / 44)$, that they completely understood explanations about side effects (69\%, 36/52 compared to $91 \%, 40 / 44)$. However, they were also less likely to report that they were in pain or discomfort (30/52) $58 \%$ compared to $71 \%(31 / 44)$.

\section{Leaving hospital}

By contrast the picture concerning discharge was less positive (Figure 4). Both groups reported problems with aspects of discharge planning. Less than $75 \%$ reported that they had been given written information, explaining the treatment and care they had received what to do when they left hospital, or that staff took their family situation into account. The proportions reporting that staff had discussed help to resume normal activities, or nursing or other support from health services was particularly low between $26 \%$ and $52 \%$.

\section{E. Clinical nurse specialists}

Breast patients were more likely to report they had been given the name of a particular clinical nurse specialist $(87 \%, 69 / 79)$ compared to lung patients $(54 \%, 37 / 68)$ and that they had contact with them (79\%, 61/77 compared to $58 \%, 37 / 64)$. Of all those who had, both breast and lung cancer patients valued care from clinical nurse specialists with $87 \%(84 / 97)$ reporting they had received answers to questions they could understand, 89\% (86/97) that they had complete confidence and trust in these nurses and $95 \%$ (92/97) that they were treated with respect and dignity by them.

\section{F. Overall care}

Reports concerning overall care reflected the marked differences so far reported. Figure 5 shows that high proportions of both groups of patients thought hospital staff had worked together and the quality of care and information provided was excellent or very good. However, slightly less than $70 \%$ of patients felt they were given emotional support during treatment always or most of the time. Lung cancer patients reported receiving information about services offering psychological or emotional support less often $(27 \%, 18 / 69)$ than breast patients $(57 \%, 43 / 75)$. They also less commonly reported having been given information about complementary therapies $(27 \%, 19 / 71$ compared to $63 \%, 48 / 76)$ or about patient support or self-help groups (27\%, 19/71 compared to $72 \%, 54 / 75)$. 
Figure 1: Finding out what was wrong with you

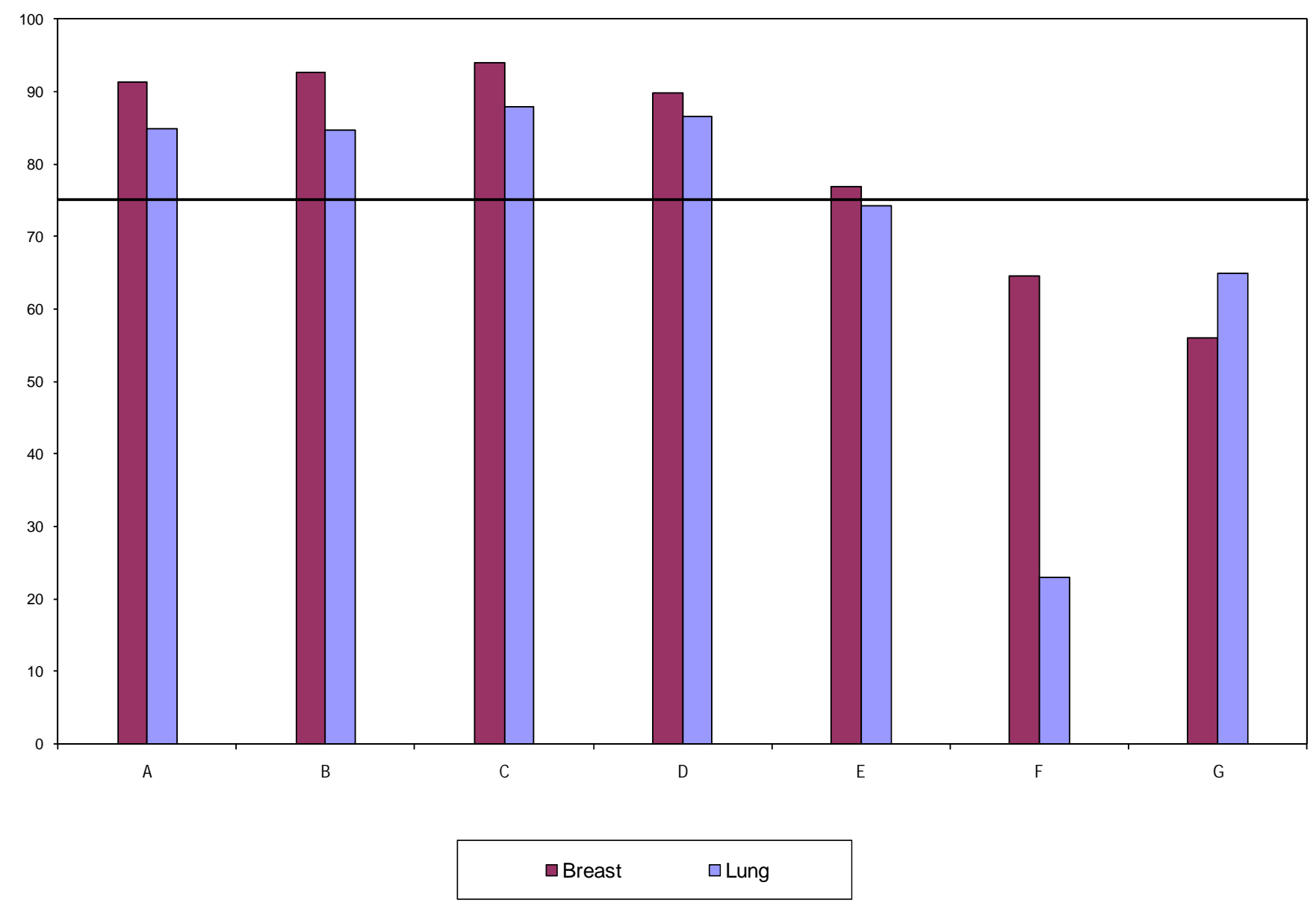
A Completely understood explanation of what was wrong
B Definitely told with sufficient sensitivity and care
$C$ If questions asked answered in a way they could understand
D Involved in discussions as much as they wanted
E Completely understood explanation of the different treatments
F CNS present
G Partner/spouse or family member present 
Figure 2: Out-patient treatment and appointments

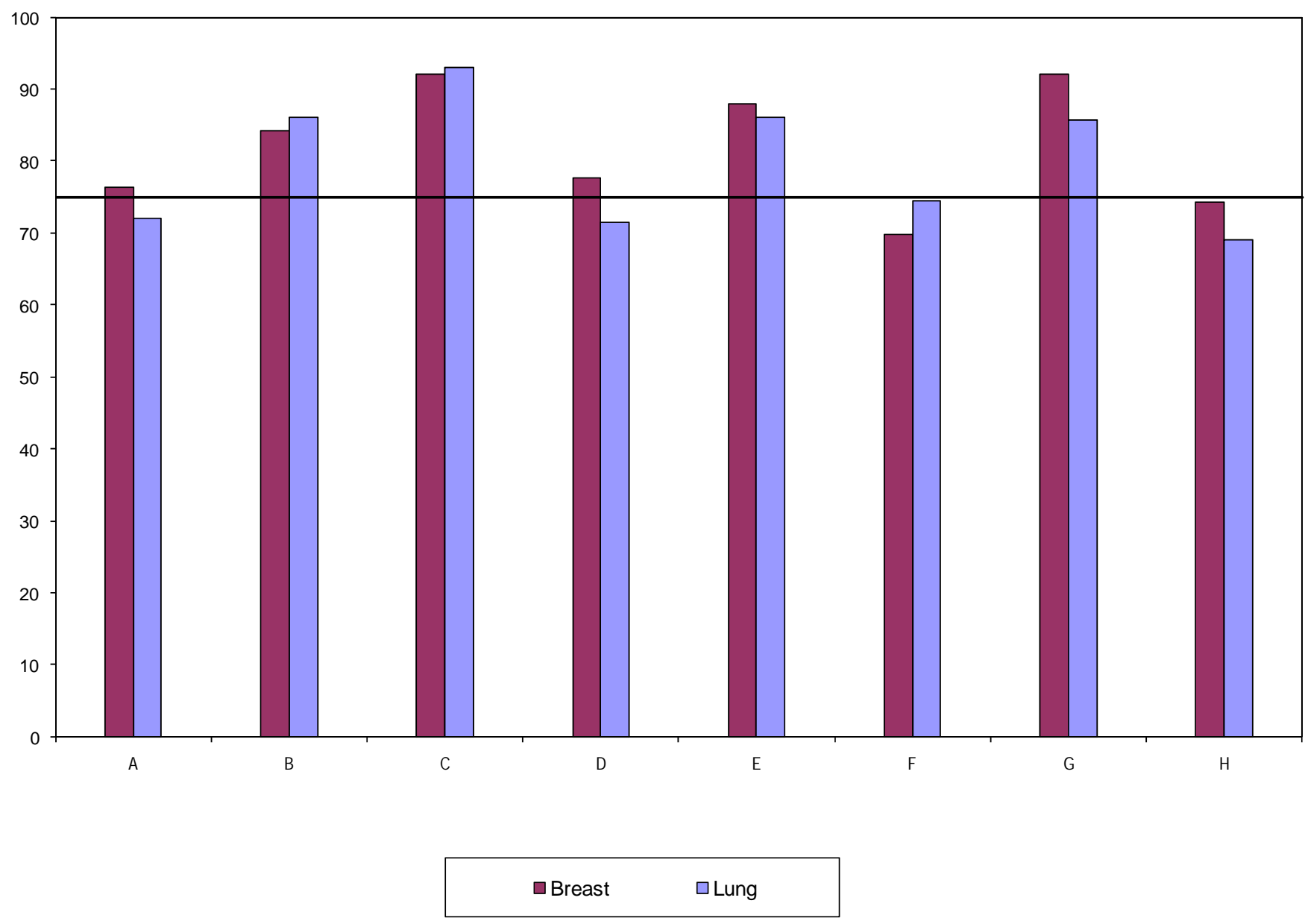
A Definitely had enough time to discuss their health or medical problem
B Complete confidence and trust
C Completely treated with respect and dignity
D Completely understood the reasons for treatment or action
E Definitely listened to what the patient said
$F$ Definitely got the answers to important questions
$G$ The doctor knew enough about their treatment or condition
$\mathrm{H}$ Given the option of receiving copies of their GP letters 


\section{Additional patient comments}

Patients' comments about what had been particularly good about their care concerned efficiency of care, the professional standard or quality of care and the emotional support and reassurance they received from staff. For example, one patient with lung cancer wrote: "I felt that all the care and treatment was of a very high standard and I am very grateful for the doctors' and nurses' professionalism. I don't think care could be improved." Patients with breast cancer made positive comments of a similar nature, but as a group they also made more suggestions for improving care (54 comments) than lung cancer patients (28 comments). For example, 16 breast cancer patients mentioned long waiting times for outpatient treatment or follow-up appointments compared to four lung cancer patients. Fourteen breast cancer patients mentioned attitudes or behaviour of some doctors and nurses, for example, lack of sympathetic or helpful staff attitudes on wards. Five breast and two lung patients commented on the need for more information including that on financial benefits or complementary therapy services available. By contrast the most common issues raised by lung cancer patients were hospital food and facilities mentioned by 10 and 6 patients, respectively. These issues and those related to the organisation of outpatient appointments were not covered by the questionnaire.

\section{Consent for data linkage}

$82 \%(67 / 82)$ of breast cancer and 87\% (65/75) of lung cancer patients consented to their clinical teams sharing information on the clinical severity of their condition with the research team to investigate whether this influenced experiences of care.

\section{Discussion}

\section{Summary of main findings}

We used an established questionnaire survey to compare the experiences of 82 breast and 75 lung cancer patients of diagnosis, overnight and day case stays and out-patient care at one Cancer Centre. Both patients groups reported similarly high levels of good experience in many areas of care, particularly in receiving their diagnosis, support from clinical nurse specialists with whom they had contact, and overall views about care. However, breast cancer patients had slightly less good experience of overnight and day case stays, while lung cancer patients received clinical nurse specialist and information about other support services after discharge less often. Both groups reported that written information was not always consistently provided at all stages. $84 \%$ of all patients consented to data on the clinical severity of their condition being used to investigate whether this influenced experiences.

\section{Comparisons to previous findings}

Our finding of good experiences around the time of diagnosis is similar to the 2005 national survey of cancer patients ${ }^{4}$. They also mirror the direction in English cancer policy which has sought to improve the quality of supportive care after diagnosis by providing health professionals with guidance and training in structuring these consultations and offering support afterwards ${ }^{20}$. The provision of written information at diagnosis has also improved nationally ${ }^{4}$. In our study $76 \%$ of breast cancer patients reported being given written information about their condition or treatment at diagnosis, compared to $64 \%$ in 2004 and 53\% in 2000 at the same centre ${ }^{5}$. Our findings also emphasise the importance of support from clinical nurse specialists, with well over $80 \%$ of patients reporting that these nurses were able to provide understandable answers to their questions, achieve their confidence and trust, and treated them with respect and dignity. These findings suggest that the information and support from a key, approachable and experienced member of staff is vital to patients' experiences of diagnosis. However, we found somewhat paradoxically that lung cancer patients, who might be expected to have greater and more immediate needs for this kind of support, were given information about and had contact with these nurses less often. Recent national census have revealed fewer clinical nurse specialists for lung cancer patients compared to those available for breast patients ${ }^{21}$. In our study lung cancer patients also appeared slightly more positive about their care than breast cancer patients. These patients may be less critical or have lower expectations, but their poor outlook and increasing prevalence of symptoms such as breathlessness with disease progression means they and their carers arguably need more rather than less support. Although we began by approaching over 100 patients each for breast and lung cancer, we could not report comparative results on a sample of at least 40 patients because not all questions were relevant to all patients. Demographic variables including age are well-established to influence experiences, but our sample size meant it was not practical to analyse these.

\section{Implications for services}

While these results show that patients in this Cancer Centre are generally reporting very positive experiences around diagnosis, a careful look at the different stages of care reveals some areas that were not performing as well. For example, shorter hospital stays, and more rapid discharge including day case surgery in breast cancer are now increasingly common place and a part of cancer policy. Our findings may indicate where teams have not yet been able to respond to the consequences of reduced stay in 
Figure 3a: Recent overnight or day case stay

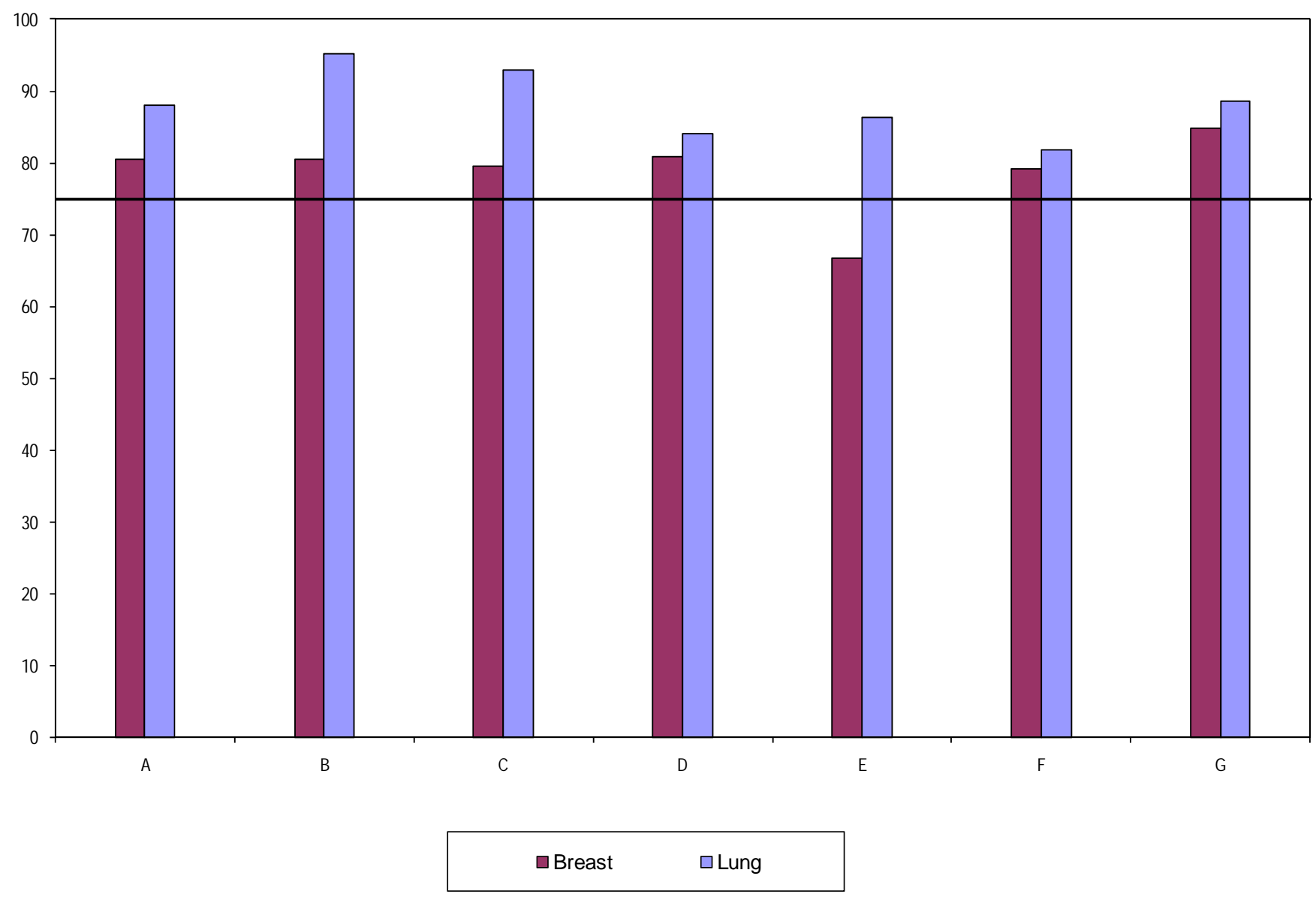
A Confidence and trust in all the hospital doctors
B Completely treated with respect and dignity by the doctors
C Always or nearly always enough doctors on duty
D Completely treated with respect and dignity by the nurses
E Always or nearly always enough nurses on duty
F Completely understood explanation of their condition
$\mathrm{G}$ Completely understood explanation of the purpose of their operation/treatment 


\section{Figure 3b: Recent overnight or day case stay}

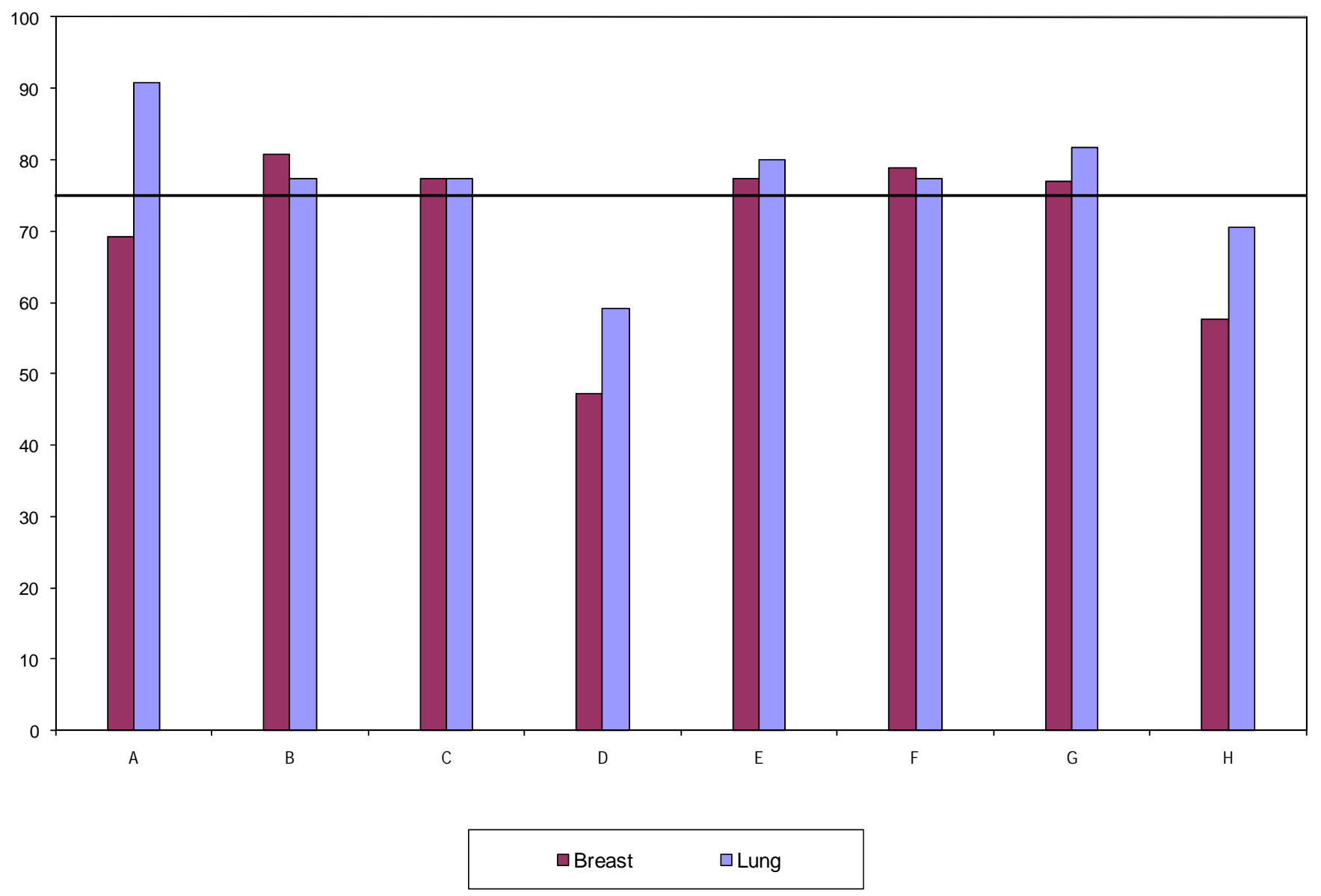
A Completely understood explanation of the possible side effects
B Never talked about their case in front of them as if they were not there
C Doctor or nurse never said one thing and another said something different
D Family and friends were involved in decisions about treatment and given the right amount of information
E Completely understood explanation of how the operation or other treatment had gone
$\mathrm{F}$ Involved in decisions about their care as much as they wanted
$G$ Given the right amount of information about the outcome of their treatment
$\mathrm{H}$ In pain or discomfort some, all or most of the time 


\section{Figure 4: Leaving hospital}

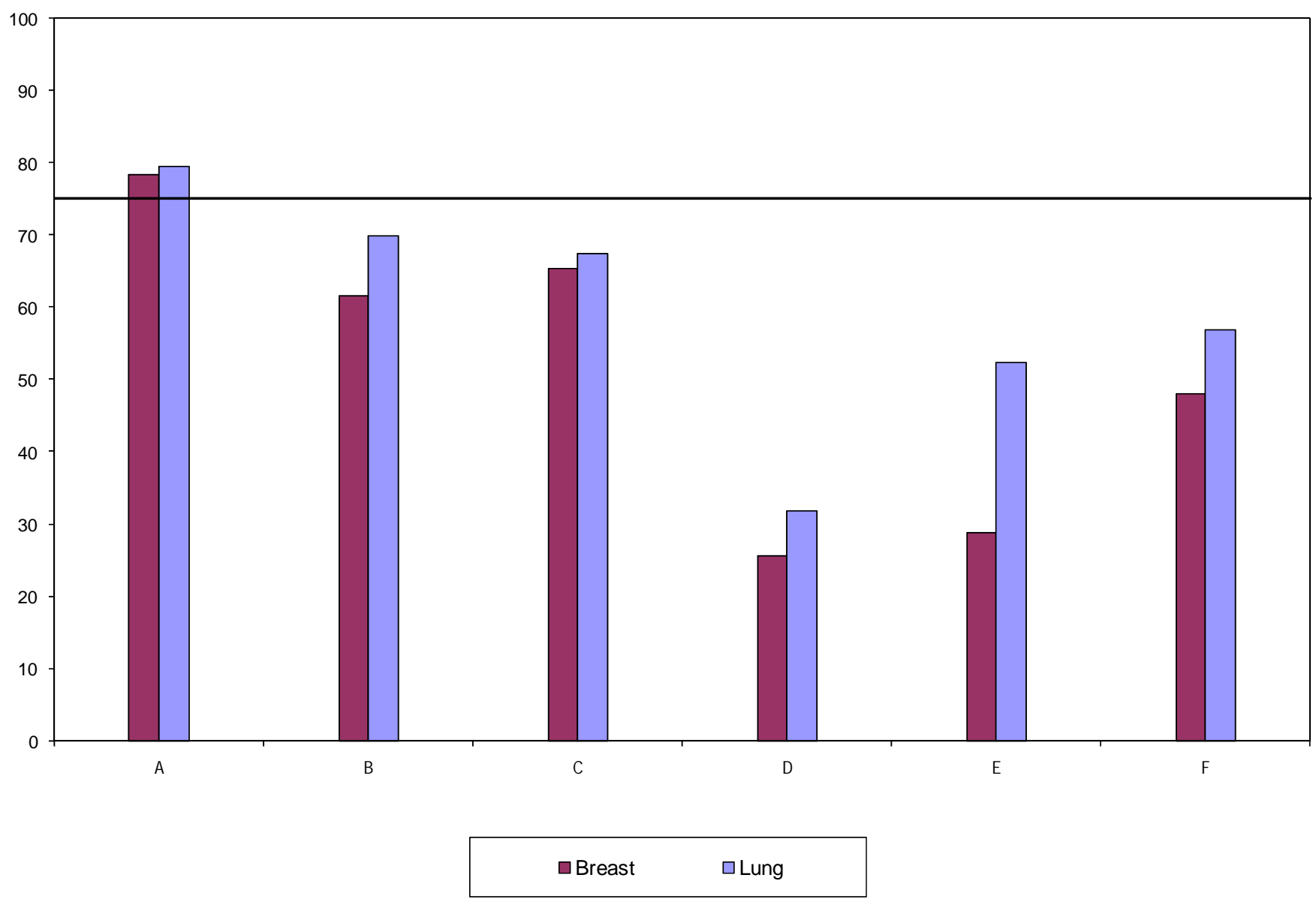

A Doctors or nurses spent enough time telling them what would happen after they left hospital

B Given written or printed information about what should do after leaving hospital

C Staff completely took their family or home situation into account

D Staff discussed additional help they would need to resume normal activities and this was provided

E Staff discussed with them whether they would need nursing or other support from health services

$\mathrm{F}$ Received a written record of the treatment/care they received 


\section{Figure 5: Overall views about care}

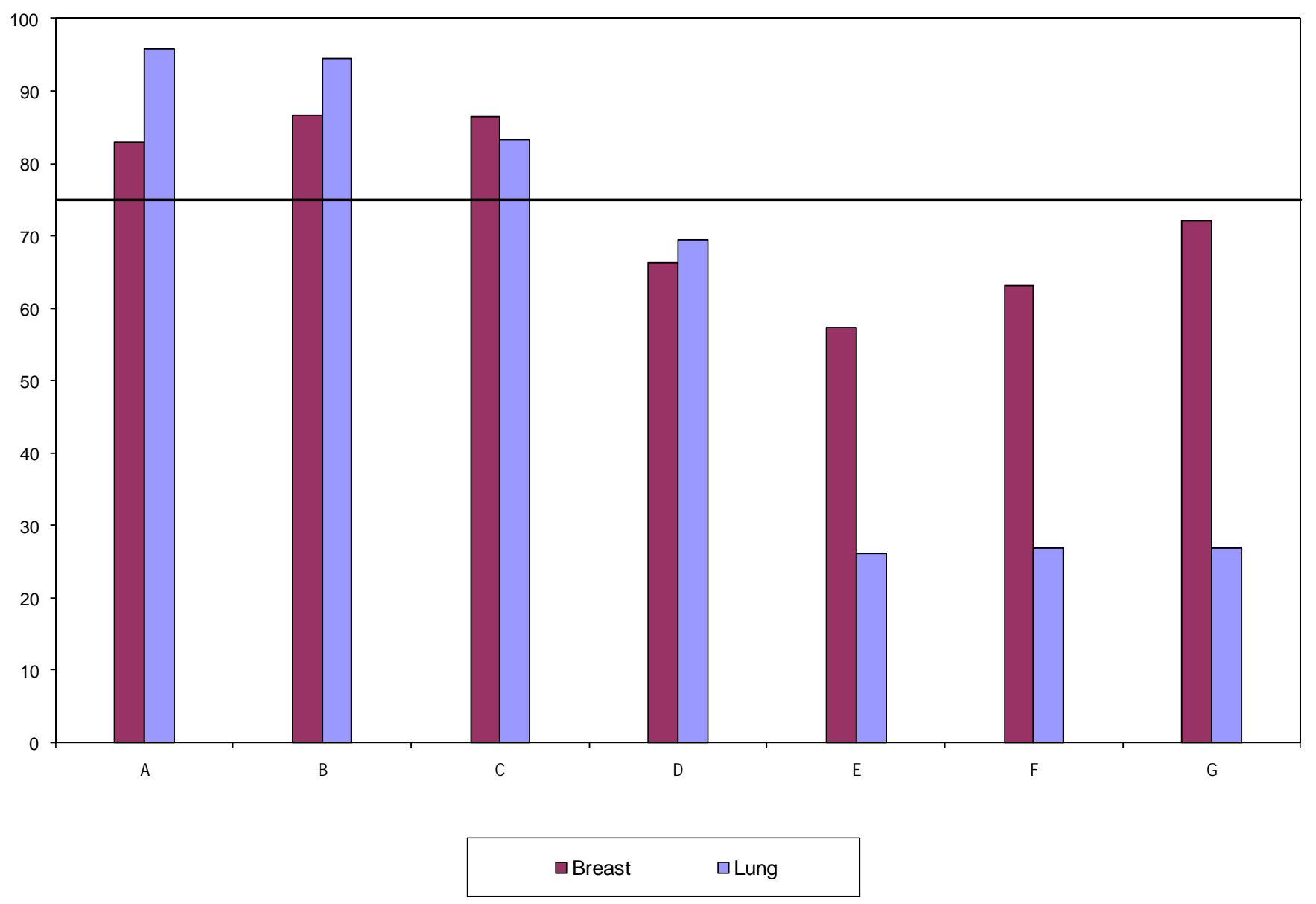
A People treating and caring for them working together always or most of the time
B Impression of quality of care and services excellent or very good
C Quality of information provided excellent or very good
D Felt given emotional support during treatment always or most of the time
E Received information about services offering psychological/emotional support
F Received information about complementary therapies
G Told about patient support or self help group 
terms of the need to give patients information and help them to self manage appropriately at home. Services will also need to investigate whether lung cancer patients see clinical nurse specialists less commonly perhaps due to a wider range of pathways to diagnosis than for breast patients and how to make this support and other information on all forms of support available to them more rapidly. Our findings show that patients were not receiving tape-recordings of their consultation despite recommendations about this. They also show that not all patients appear to want to have someone present when they are told their diagnosis, suggesting that preferences should be ascertained rather than assumed by health professionals.

Experience of conducting this survey suggests that future services can expect good survey responses from patients with lung cancer facing a poor outlook if they are not excluded, and surveys are sent in a timely manner shortly after diagnosis and in an individualised way. It is also important for services to consider tailoring the questions to focus on specific items that are seen as relevant to local services and can produce specific information relevant for quality improvement. That some of the questions were sensitive enough to detect differences both between two local services and over time suggests that other services should consider this strategy. In our service it revealed pertinent issues for patients on which improvement should focus.

\section{Implications for policy}

Current health policies in developed countries [1,6,7,9] place renewed emphasis on collecting and making publically available information on patients' experiences of care and their care outcomes. Our findings show the usefulness of survey data from a comparative patient group, and therefore how useful bench marking data on experiences of patients in different cancer centres in the ongoing national surveys would be. We decided to focus on results where less than $75 \%$ of patient reported a good experience, but this is an arbitrary threshold that still means one in four patients are reporting a less good experience. A consensus over whether this is acceptable might be developed as services develop skill in using and responding to patient survey data. Although we had generous local funding for this survey, data collection, quality assurance and processing of patient and survey data turned out to be a complex and time-consuming task. The benefits of benchmarked data and the possibility of economies of scale suggest to us that routine annual surveys are best conducted from a central point as has been the case in US organisations like the Veterans Health Administration which have many years experience of their use [22,23].

\section{Implications for research}

Despite some initial concern that the survey might be a burden to the more ill patients, we obtained a good response rate from patients with lung cancer facing a poor prognosis. The willingness that both breast and lung cancer patients indicate to give consent for their data to be linked to other clinical data on disease severity means that with larger samples it might be possible to determine whether experiences are associated with differing disease stage and other case mix variables. For example, a recent study of US Veterans has suggested that patients admitted after myocardial infarction who reported better experiences of care were also at a modest decreased risk of dying one year later ${ }^{24}$. The data linkage programme of the US National Cancer Institute has also demonstrated that a wide range of studies exploring differences in health-related quality of life for older patients and the impact of different cancers on patients are now practical $[25,26]$. These models could be used to explore similar questions using existing national datasets in other countries.

\section{Acknowledgements}

We thank the patients who helped with the pilot of the questionnaire and took part in the survey for their time and thoughts.

We thank North London Cancer Network and The Picker Institute for allowing us to use their questionnaire and the Patient Centred Care Project Team and clinical staff for helping to adapt and pilot the questionnaire.

We thank hospital trust staff for providing patient data and Thames Cancer Registry staff for computing and administrative support.

The findings upon which this paper are based are drawn from the Integrated Cancer Centre's 'Patient Centred Care’ project; a collaboration between Guy’s \& St Thomas' NHS Foundation Trust, King's College Hospital NHS Foundation Trust and King's College London in partnership with The King's Fund. The project was funded by Guy's \& St Thomas' Charity.

This work was carried out by Thames Cancer Registry, King's College London which receives funding from the Department of Health; the views expressed in this publication are those of the authors and not necessarily those of the Department of Health.

\section{References}

[1] Shaller, D. (2007) Patient-Centered Care: What does it take? Commonwealth Fund: New York. http://www.commonwealthfund.org/Content/Publications/FundReports/2007/Oct/Patient-Centered-Care--What-Does-ItTake.aspx (Last accessed 30 January 2011) 
[2] Coulter A., Fitzpatrick R. \& Cornwell J. (2009) The Point of Care. Measures of patients' experience in hospital: purpose, methods and uses. The King's Fund, London. http://www.kingsfund.org.uk/publications/measures.html (Last accessed 30 January 2011)

[3] Department of Health (2002) National Survey: Cancer Patients.

http://www.dh.gov.uk/en/Publicationsandstatistics/PublishedSurv ey/NationalsurveyofNHSpatients/Nationalsurveycancer/index.ht m (Last accessed 30 Jan 2011)

[4] National Audit Office (2005) Tackling cancer: Improving the patient journey. Department of Health. The Stationery Office: London.

http://www.nao.org.uk/publications/0405/tackling_cancer.aspx (Last accessed 30 Jan 2011)

[5] Madden P.B. \& Davies E.A. (2010) Reporting cancer patients' experiences of care for quality improvement: analysis of 2000 and 2004 survey results for South East England. Journal of Evaluation in Clinical Practice 16, 776-783

[6] Department of Health (2010) Equity and Excellence: Liberating the NHS. White paper. http://www.dh.gov.uk/en/Publicationsandstatistics/Publications/P ublicationsPolicyAndGuidance/DH_117353 (Last accessed 30 Jan 2011)

[7] Department of Health (2010) Cancer patient experience. Surveys of patient experience: survey report 2010. http://www.dh.gov.uk/en/Healthcare/Cancer/Patientexperience/in dex.htm (Last accessed 30 Jan 2011)

[8] Armes J., Crowe M., Colbourne L., Morgan H., Murrels T., Oakley C., Palmer N., Ream E., Young A. \& Richardson A. (2009) Patients' supportive care needs beyond the end of cancer treatment: a prospective, longitudinal survey. Journal of Clinical Oncology 27 (36), 6172-6179

[9] Howell D., Bakker D., Doran D., Fitch M., Green E., Sussman J., Chulak T. \& Chundamala J (2010) Patient-focused Cancer Care Outcomes: A core set of validated patient-reported measures for Canada. Canadian Institutes of Health Research May 2010

[10] Department of Health (2007) Cancer Reform Strategy Department of Health Publications: London. http://www.dh.gov.uk/en/Publicationsandstatistics/Publications/P ublicationsPolicyAndGuidance/DH_081006 (Last accessed 30 Jan 2011)

[11] McCarthy M., Datta P., Sherlaw-Johnson C., Coleman M. \& Rachet B. (2008) Is the performance of cancer services influenced more by hospital factors or by specialization? Journal of Public Health 30 (1), 69-74

[12] Ambs A., Warren J.L., Belizzi K.M., Topor M., Haffer S.C. \& Clauser S.B. 2008 Overview of the SEER - Medicare Health Outcomes Survey linked dataset. Health Care Financing Review 29 (4), 5-21

[13] Davies E. \& Cleary P. (2005) Hearing the patient's voice? Factors affecting the use of patient survey data in quality improvement. Quality and Safety in Health Care 14, 428-432

[14] Reeves R. \& Seccombe I. (2008) Do patient surveys work? The influence of a national survey programme on local qualityimprovement initiatives. Quality and Safety in Health Care 17, 437-441

[15] Picker Institute Europe (May 2009) Executive Summary. North London Cancer Network Patient Survey 2008. http://www.pickereurope.org/Filestore/PIE_reports/Survey_report s/North_London_Cancer_Network_Patient_Survey_2008_ExecS ummary2.pdf (Last accessed 30 January 2011)
[16] Picker Institute Europe (2008) Patient experience surveys: the rationale.

http://www.pickereurope.org/Filestore/Misc/Survey_theory_for_ web.pdf (Last accessed 30 January 2011)

[17] National Cancer Action Team (2008) National Cancer Peer Review Programme. Manual for Cancer Services 2008: Breast Measures. Available at:

http://www.cquins.nhs.uk/?menu=resources (Last accessed 30 January 2011)

[18] Edwards PJ, Roberts I, Clarke MJ, DiGuiseppi C, Wentz R, Kwan I, Cooper R, Felix LM, Pratap S. Methods to increase response to postal and electronic questionnaires. Cochrane Database of Systematic Reviews 2009, Issue 3. Art. No.: MR000008. DOI: 10.1002/14651858.MR000008.pub4

[19] The English Indices of Multiple Deprivation (2007) Communities and Local Government, March 2008. http://www.communities.gov.uk/publications/communities/indici esdeprivation07 (Last accessed 30 January 2011)

[20] National Institute for Clinical and Public Health Excellence (2004). Supportive and Palliative Care Guidance: The Manual.

http://guidance.nice.org.uk/CSGSP/Guidance/pdf/English (Last accessed 30 January 2011)

[21] Trevatt P. \& Leary A. (2010) A census of the advanced and specialist cancer nursing workforce in England, Northern Ireland and Wales European Journal of Oncology Nursing 14 (1), 68-73

[22] Perlin J.B., Kolodner R.M. \& Roswell R.H. (2004) The Veterans Health Administration: quality, value, accountability and information as transforming strategies for patient-centered care. American Journal of Managed Care 10 (part 2), 828-836

[23] Wright S.M., Craig T., Campbell S., Schaefer J. \& Humble C. (2006) Patient satisfaction of female and male users of Veterans Health Administration services. Journal of General Internal Medicine 21 March Supplement 3, S26-32

[24] Meterko M., Wright S., Lin H., Lowry E. \& Cleary P.D. (2010) Mortality among Patients with Acute Myocardial Infarction: The Influences of Patient-Centered Care and Evidence-Based Medicine. Health Services Research: 45 (5), 1188-1204

[25] Clauser S.B., Arora N.K., Bellizzi K., Haffer S.C., Topor . \& Hays R.D. (2008) Disparities in HRQOL of cancer survivors and non-cancer managed care enrollees. Health Care Financing Review 29 (4), 23-40

[26] Reeve B.B., Potosky A.L., Wilder Smith A., Han P.K., Hays R.D., Davis W.W., Arora N.K., Haffer S.C. \& Clauser S.B. (2009) Impact of cancer on health-related quality of life in older Americans. Journal of the National Cancer Institute, 101 (12), 860-868 